\title{
Harmonic generation in organ pipes, recorders, and flutes
}

\author{
N. H. Fletcher and Lorna M. Douglas
}

Department of Physics, University of New England, Armidale New South Wales 2351, Australia (Received 25 September 1979; accepted for publication 6 March 1980)

\begin{abstract}
A simplified treatment is given of the mechanism of sound production in musical instruments driven by air jets, which is sufficiently explicit that semiquantitative predictions can be made about the effects of certain variables upon the harmonic structure of the sound produced. In particular it is found that the amplitude of the even harmonics, generally, and of the second harmonic, particularly, is quite critically dependent upon the offset of the pipe lip from the symmetry plane of the jet. A completely symmetrical relationship (zero offset) reduces the generated amplitude of the second harmonic by a large factor. Experimental results with an adjustable organ pipe are found to confirm these predictions. The implications of these results for the voicing of organ pipes and recorders and for subtle tonal variation in flute playing are briefly discussed.
\end{abstract}

PACS numbers: $43.75 . \mathrm{Np}$

\section{INTRODUCTION}

The mechanism of sound production by flue pipes, under which heading are included organ flue pipes, recorders (German: Blockflöte), and flutes, is now fairly well understood, ${ }^{1-7}$ not only in its general principles but also in relation to generation of the harmonic spectrum of the sound produced $d^{4,6}$ and the characteristic attack transient. ${ }^{7}$ The full theory of harmonic generation is complicated $d^{6,9}$ and involves solution of a large number of coupled nonlinear differential equations, ${ }^{6}$ or their related integral equations ${ }^{9}$ so that the essential features of the mechanism are somewhat obscured. It turns out to be possible, however, to deduce a simplified version of the theory which accounts well for the behavior of the pipe and the effects of several voicing or playing adjustments and which is free from all the mathematical elaboration of the detailed treatment. It is the purpose of this paper to outline and justify this simplified approach, to set out its predictions, and to compare these with experimental measurements for several cases of interest. In this way we arrive at a straightforward and semiquantitative understanding of several important features of pipe voicing adjustments and their effect on tone quality.

\section{THEORETICAL PRINCIPLES}

The sounding mechanism of flue pipes has been discussed in detail elsewhere ${ }^{6}$ and a recent review ${ }^{10}$ sets out the principles in a concise way. Briefly, and referring to Fig. 1, an air jet emerges from the flue (which is a fixed structure in an organ pipe or recorder

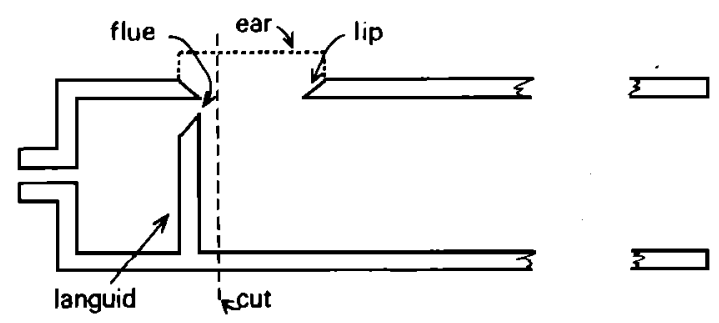

FIG. 1. The geometry of a typical organ flue pipe. For our experiments the pipe was cut along the plane shown and the body moved relative to the foot and flue. and is the opening in the player's lips in the case of the flute), travels some distance across the mouth of the pipe, and then interacts with the pipe lip. The acoustic flow out of the pipe mouth associated with the vibration of the pipe air column induces waves on the jet which grow in amplitude as they propagate so that the transverse motion of the jet at the pipe lip is generally comparable with the jet width when the pipe is sounding normally. The jet blowing alternately into and outside the pipe at its lip maintains the pipe oscillation so that steady sound results.

Careful consideration ${ }^{5.6}$ shows that the driving force exerted on the pipe modes by the deflecting jet has terms proportional, respectively, to the volume flow of the jet into the pipe and to the square of this quantity, but under most conditions the simple volume flow term is dominant. Phase relationships are a little complex ${ }^{6,10}$ but this need not concern us here. If the jet velocity is such that there is just half a wavelength of the transverse disturbance between the flue and the lip, then the pipe will sound exactly at its resonance frequency. If the blowing pressure is increased to shorten the jet travel time then the sounding frequency will rise slightly to introduce a compensating phase shift, while if the blowing pressure is lowered the sounding frequency will fall slightly.

Harmonic generation occurs primarily because the jet velocity has a bell-shaped profile so that, even if the jet is being deflected sinusoidally, its flow into the pipe will be a distorted sinusoid containing higher harmonic components whose frequencies approximate those of higher resonant modes of the pipe. There is a small additional upper harmonic component generated by a Bernoulli term in the flow interaction ${ }^{5.6}$ but this is of small amplitude in a normal pipe configuration. ${ }^{6}$

In our simplified development of the theory we consider only the upper harmonics generated by the jet profile, thus computing what might be called a "source spectrum" which is then modified by interaction with the array of not-exactly harmonic resonances of the pipe, treated as a "filter function." This is the approach originally advocated by Sundberg, ${ }^{11}$ though he was forced to assume rather than to derive an ap- 
.propriate source spectrum. The method cannot be justified for a general case, since in principle each harmonic interacts back upon the jet to modify the source spectrum, but in the present case it represents a good approximation. There are several reasons for this: (1) for many flue pipes of practical interest, the fundamental is the strongest component of the radiated sound; (2) the internal velocity spectrum of the pipe is further weighted towards low frequencies at $6 \mathrm{~dB}$ per octave relative to the radiated spectrum because of the relation between source strength and radiated sound pressure; (3) the internal acoustic displacements (which are what really concern us in calculating jet displacement) have in addition the further low-frequency emphasis of $6 \mathrm{~dB}$ per octave arising from the fact that displacement is the integral of velocity. The exceptions to these generalizations occur only for the lowest notes of the Boehm flute and for organ flue pipes of "string" quality, where the fundamental is relatively weak. Thus, to a good approximation, the displacement of the jet at the pipe lip is simply sinusoidal, with frequency equal to and amplitude proportional to those of the pipe fundamental, and we can proceed to calculate the source spectrum by examining the waveform of the resulting jet flow into the pipe.

\section{JET SOURCE FUNCTION}

For a symmetrical planar jet in the laminar flow regime at low Reynolds' numbers the velocity profile has the form ${ }^{12}$

$$
V(y)=V_{0} \operatorname{sech}^{2}(y / b),
$$

where $y$ is the distance from the axial plane, $V_{0}$ is the central velocity, and $b$ is a scale factor determining the width of the profile (see Fig. 2). As the jet spreads downstream from the flue $V_{0}$ decreases and $b$ increases.

The jets in real musical flue pipes are rarely in the laminar flow regime but are usually homogeneously turbulent. The nicking on the languids of some organ pipes perhaps serves to establish this homogeneity. The behavior of a turbulent jet is, however, quite similar to that of a laminar jet as far as its function in a flue pipe is concerned, and indeed the jet divergence and wave propagation behavior is considerably simp-

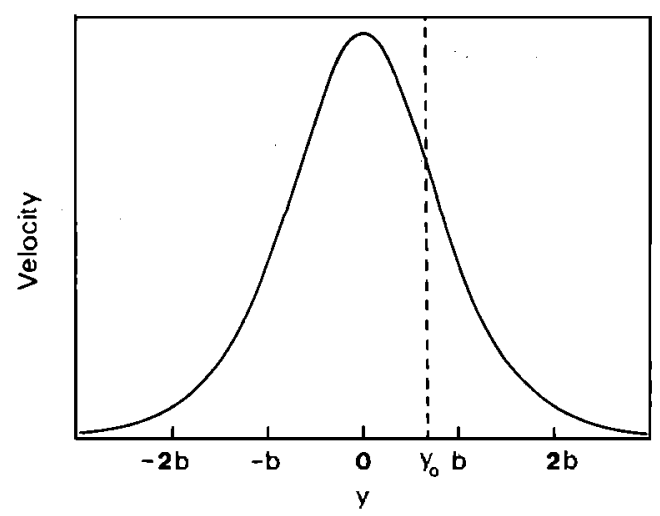

FIG. 2. Axial velocity distribution across a laminar jet as given by (1). The mean axial distribution across a turbulent jet is very similar. ler. ${ }^{13.14}$ The mean velocity profile of a turbulent jet does not have exactly the form.(1), but this function is a sufficiently close approximation to serve our purpose and has the advantage of algebraic simplicity.

Referring to Fig. 2, where the function (1) is plotted, suppose that the pipe lip cuts the jet at the position $y_{0}$ when in equilibrium, and that the jet then oscillates in the $y$ direction with amplitude $a$ and angular frequency $\omega$. Then the jet flow entering the pipe has the form,

$$
\begin{aligned}
U(t) & =\int_{-\infty}^{y_{0}+a \sin \omega t} V(y) d y \\
& =V_{0}\left\{1+\tanh \left[\left(y_{0}+a \sin \omega t\right) / b\right]\right\} .
\end{aligned}
$$

Clearly, $U(l)$ is not sinusoidal but contains harmonics of all orders, that with frequency $n \omega$ having amplitude

$$
\left.U_{n}=(\omega / \pi) \mid \int_{0}^{2 \pi / \omega} U(t) \frac{\sin }{\cos }\right\} n \omega t d t \mid,
$$

where the sine is used for odd $n$ and the cosine for even $n$. $U_{n}$ clearly depends both on the lip offset $y_{0}$ and on the amplitude $a$.

It is possible also to see the qualitative form of the variation of the $U_{n}$ with $y$ by expanding $U$ as a function of $y_{0}$ in a Taylor's series about $y=y_{0}$. Recalling that $\sin ^{n} \omega t$ can be written ${ }^{15}$ as a sum of terms in either $\sin (n-2 m) \omega t$ or $\cos (n-2 m) \omega t$ for $m=0,1,2, \ldots$, and keeping just leading terms, which is appropriate for $a / b \ll 1$, then,

$$
U_{n} \propto(a / b)^{n}\left|d^{n} U / d y^{n}\right|_{y=y_{0}} \propto(a / b)^{n}\left|d^{n-1} V / d y^{n-1}\right|_{y=y_{0}} .
$$

The immediately interesting observation is that the even harmonics are all identically zero when the lip is symmetrically placed in relation to the jet $\left(y_{0}=0\right)$ while the odd harmonics are maximal in this configuration. This effect was already noted in the earlier complete treatment ${ }^{4}$ but now emerges, as we see later, as one of the most important practical consequences of voicing or playing adjustments. The other result worthy of comment is that, from (4), the amplitude of the $n$th harmonic varies as the $n$th power of the amplitude of the fundamental, a result derived for a somewhat different case by Worman. ${ }^{16}$

As we have already remarked, the amplitude $a$ of jet displacement at the lip is not generally small compared with the jet scale width $b$ at this point, so that we need to calculate the full expression (3) for the Fourier components of the excitation spectrum for $a / b \sim 1$ in order to compare the predictions of the theory realistically with experiment. These calculations are shown in Fig. 3 from which it can be seen that, while the qualitative conclusions about even harmonics remain unaltered, the variation of the odd harmonics now shows a plateau region around the symmetrical configuration $y_{0}=0$. We conclude that lip adjustment is not critical to the low-numbered odd harmonics but may be critical to the even harmonics.

Before turning from theory to experiment we should note several additional points. Firstly what we have 


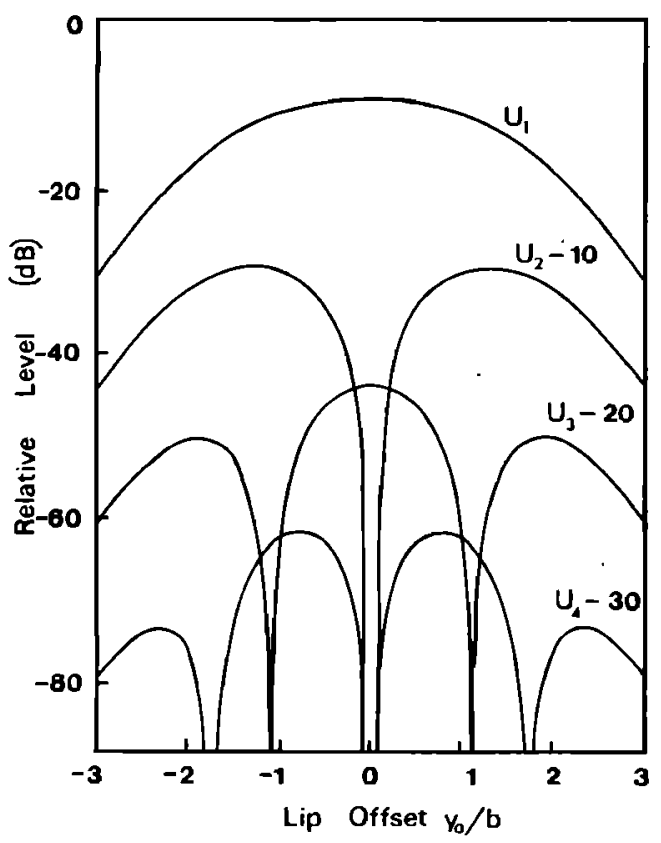

FIG. 3. Calculated relative pressure levels of the first four harmonics in the jet source function as functions of the relative lip displacement $y_{0} / b$, as given by (2). The relative jet displacement amplitude is $a / b=2$. For clarity the curves for successive harmonics are displaced downwards by 10,20, and $30 \mathrm{~dB}$ relative to the scale for the first harmonic.

calculated is the internal source spectrum of the jet and this must be modified both by the filter function of the pipe resonator and by the radiation function of its open ends in order to derive the radiated spectrum. These together, determine the absolute level of each harmonic but do not depend on the lip offset voicing adjustment $y_{0}$. We should thus be able to compare measured sound output curves with the calculated curves of Fig. 3 if absolute levels are ignored. Secondly, we have left out of consideration the small amount of second harmonic (and thence of other even harmonics), generated by the Bernoulli nonlinearity of the jet-drive mechanism. ${ }^{5}$ Inclusion of this effect would simply mean a small component proportional to $a^{2}$ and independent of $y_{0}$ added to $U_{2}$, and similarly for the higher even harmonics. This would change the zeros shown in Fig. 3 into simple minima. Finally, we should point out that the lip offset $y_{0}$ is measured from the center plane of the undisturbed jet at the lip position. As discussed elsewhere ${ }^{6}$ there is generally a small static displacement of the jet caused by static pressure increase within the pipe, so that experimental determination of $y_{0}$ may not be simple.

\section{ORGAN PIPE EXPERIMENT}

To examine experimentally the predictions of this theory we took an adjustable flute-type organ pipe used in a previous study ${ }^{6}$ and having a square cross section about $40 \times 40 \mathrm{~mm}$ and length $460 \mathrm{~mm}$ giving a fundamental near $330 \mathrm{~Hz}$. Pipe material was Perspex $6 \mathrm{~mm}$ thick and the languid was of the type known to organ builders as "inverted" with a $60^{\circ}$ bevel inside the foot of the pipe. For the present measurements "ears" were added and the pipe was cut through just above the languid as suggested by Nolle ${ }^{17}$ so that the foot and flue could be moved backwards and forwards relative to the lip and pipe body, thus varying the offset $y_{0}$. This motion was effected by a screw connected to a multi-turn potentiometer to provide displacement along one axis of an XY plot, the other coordinate being provided by the appropriately filtered output of a microphone mounted on the axis of the pipe, about $0.5 \mathrm{~m}$ from the open end, in an anechoic room. By plotting the sound pressure logarithmically, the experimental results can thus be compared directly with the theoretical results of Fig. 3.

We should note that some of the results detailed below have already been measured by Nolle, ${ }^{17}$ though in a less systematic fashion since he did not have the predictions of an explicit theory to guide him in determining what parameters are important.

This experimental arrangement behaved consistently for a considerable range of blowing pressures, flue widths and lip cut-up distances. Figure 4 shows the results of one of the better measurements which is, however, quite typical of all those obtained. There is clearly quite good semiquantitative agreement with the theoretical curves of Fig. 3, if $a / b$ is assumed to have a value near 2, which is quite reasonable. More importantly, however, the experimental curves exhibit very clearly the minima in the even harmonics, and specially the second harmonic, expected from the theory. This feature is important not only as a test of the theory but also because variation in the intensity of the second harmonic of a complex tone is a very effective means of achieving a variation in tone color, such variations supplying a large measure of the difference between open and stopped organ pipes or,

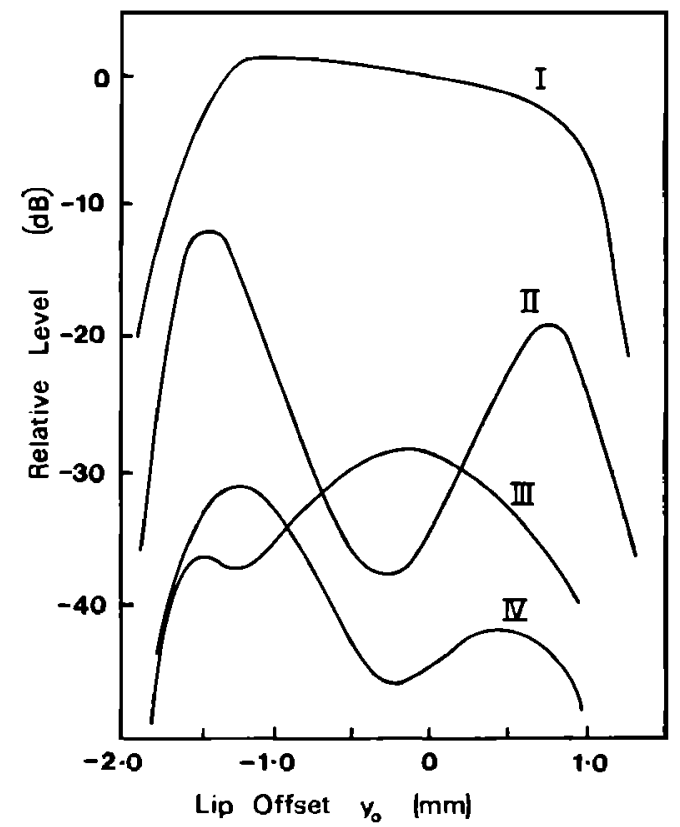

FIG. 4. Measured relative sound pressure levels of the harmonics in the radiated sound of the experimental pipe for a cut up of $10 \mathrm{~mm}$, a flue width of $1 \mathrm{~mm}$, and blowing pressure of $50 \mathrm{~Pa}$, as functions of lip offset $y_{0}$ from the nominally symmetric position. (100 $\mathrm{Pa}=1 \mathrm{~cm}$ water gauge.) 
among reed-driven instruments, between low-register notes on the oboe and the clarinet. As expected, the even harmonics do not go strictly to zero, partly because of the omitted nonlinear term in the jet drive and partly, no doubt, because of incomplete symmetry in the actual jet flow and lip shape. This also probably accounts for the slight asymmetry of the whole set of curves.

By examination of acoustic waveforms on an oscilloscope we readily see that the phase of the second harmonic changes by $180^{\circ}$ across the minimum at $y_{0}=0$. This is just what is expected from the theoretical result (4) if the algebraic rather than the absolute value of the derivative is considered.

Finally, we examined the effects of the sharpness of the upper lip of the pipe by beginning with a sharp wedge of about $30^{\circ}$ angle and then squaring or rounding it off to a thickness of up to about $3 \mathrm{~mm}$. These changes made no difference to the absolute sound pressure levels of the harmonics or to their behavior with lip offset, though more subtle characteristics like background air noise or onset transient might well have been influenced.

\section{THE RECORDER}

Members of the recorder or Blockflöte family are essentially built like organ flue pipes except that the windway below the flue is long and narrow (typically $10 \mathrm{~mm} \times 2 \mathrm{~mm}$ in cross section and $60 \mathrm{~mm}$ in length for an alto recorder) and the tapered body is provided with finger holes. The lip cut-up is small (3 to $4 \mathrm{~mm}$ ) and the lip is arranged nearly centrally in relation to the flue opening (so $y_{0} \approx 0$ ). From our previous discussion we should therefore expect the even harmonics in recorder sound to be weak relative to the odd harmonics and indeed this effect, which gives to recorders of good quality their characteristic "hollow" sound, has been previously noted by Lupke ${ }^{18}$ and by Herman, ${ }^{19}$ though attributed by Herman for some reason to the slight conicity of the bore.

In a set of measurements ${ }^{20}$ taken some time ago in this laboratory we followed the development of individual harmonics in the sound of a typical recorder of moderate quality as a function of blowing pressure. Figure 5, extracted from these results, is fairly typical and shows the nearly independent behavior of the odd and even harmonics, as well as the low relative level of the even harmonics. This behavior is rather what we should expect from our simple theory, while the independent coupling of odd and even harmonics follows in greater detail as a consequence of a nonlinearity in which the cubic term is large compared with the quadratic term. ${ }^{6}$

\section{THE FLUTE}

In the normal transverse orchestral flute, the player's lips take the place of the fixed flue of the organ pipe and direct a jet of air against the sharp edge (in-

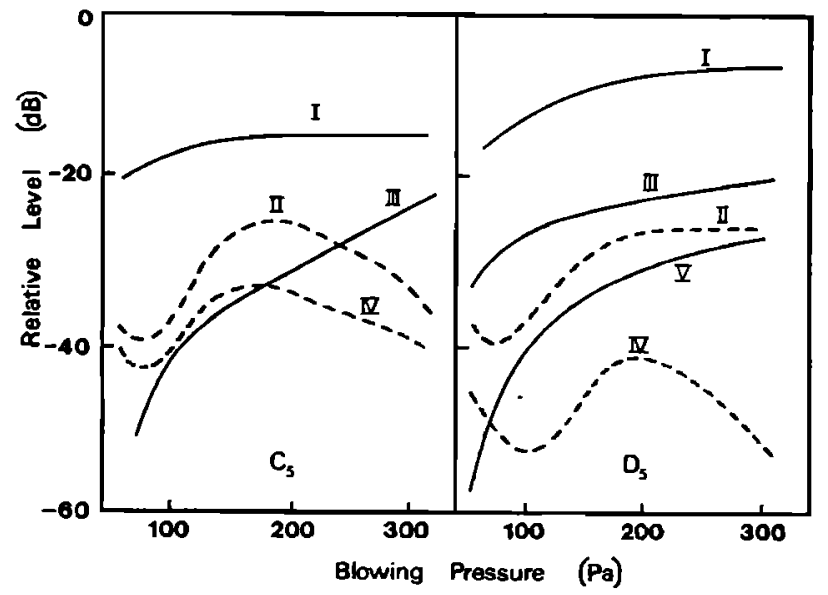

FIG. 5. Measured relative sound pressure levels of the harmonics for the notes C5 $(523 \mathrm{~Hz})$ and D5 $(587 \mathrm{~Hz})$, as a function of blowing pressure, played on an alto recorder by Schreiber. Both notes have a pleasantly rich sound.

cluded angle about $80^{\circ}$ ) of the embouchure hole. The jet geometry is under the continuous control of the player, as also is the blowing pressure. ${ }^{21}$ Such subtle adjustment possibilities make any objective study of tone quality in relation to jet geometry extremely difficult, but it is noteworthy that good flute players are able to produce significant changes in tone color by changing minor aspects of jet geometry and blowing pressure. In the light of our discussion above it seems likely that one of the most important quantities accessible to the player may be the jet direction, by adjustment of which he can change the relative intensity of the second harmonic. A qualitative experiment suggests that this is indeed so, but measurement is made difficult by the normal presence of vibrato caused by rhythmically varying blowing pressure. ${ }^{21}$

\section{CONCLUSIONS}

The simple theory presented above enables us to understand, in a semiquantitative way, the mechanism of harmonic production in flue pipes. We have essentially dealt only with the source function for the interaction of the jet with the pipe lip. Upon this must be superposed the highly selective filtering action of the pipe resonator as discussed by Sundberg ${ }^{11}$ and by Benade. ${ }^{22}$

A result of major importance to understanding the effect of voicing adjustments in organ pipes and recorders and of tone variation in flute playing is the theoretical prediction, confirmed experimentally, that the amplitudes of the even harmonics generally and of the second harmonic specifically depend quite critically upon the offset of the pipe lip relative to the center plane of the jet.

\section{ACKNOWLEDGMENT}

This study is part of a program in musical acoustics supported by the Australian Research Grants Committee. 
${ }^{1} \mathrm{~L}$. Cremer and H. Ising, "Die selbsterregten Schwingungen von Orgelpfeifen," Acustica 19, 143-153 (1967).

${ }^{2} \mathrm{~J}$. W. Coltman, "Sounding mechanism of the flute and organ pipe," J. Acoust. Soc. Am. 44, 983-992 (1968).

${ }^{3} \mathrm{~S}$. A. Elder, "On the mechanism of sound production in organ pipes," J. Acoust. Soc. Am. 54, 1554-1564 (1973).

"N. H. Fletcher, "Non-linear interactions in organ flue pipes," J. Acoust. Soc. Am. 56, 645-652 (1974).

${ }^{5} \mathrm{~N}$. H. Fletcher, "Jet-drive mechanism in organ pipes," J. Acoust. Soc. Am. 60, 481-483 (1976).

${ }^{6}$ N. H. Fletcher, "Sound production by organ flue pipes," J. Acoust. Soc. Am. 60, 926-936 (1976).

${ }^{2} \mathrm{~N}$. H. Fletcher, "Transients in the speech of organ flue pipes-a theoretical study," Acustica 34, 224-233 (1976).

${ }^{8} \mathrm{~N}$. H. Fletcher, "Mode locking in non-linearly excited inharmonic musical oscillators," J. Acoust. Soc. Am. 64, 15661569 (1978).

${ }^{9} \mathrm{R}$. T. Schumacher, "Self-sustained oscillations of organ flue pipes: An integral equation solution," Acustica 39, 225-238 (1978).

$10 \mathrm{~N}$. H. Fletcher, "Air flow and sound generation in musical wind instruments," Ann. Rev. Fluid Mech. 11, 123-146 (1979).

"J. Sundberg, "Mensurens betydelse i öppna labialpipor" (with English summary), Acta Univ. Ups., Stud. musicol. Ups. (N.S.) 3, 1-224 (1966).
12W. G. Bickley, "The plane jet," Philos. Mag. 28, 727-731 (1937).

${ }^{13}$ N. H. Fletcher and S. Thwaites, "Wave propagation on an acoustically perturbed jet," Acustica 42, 323-334 (1979).

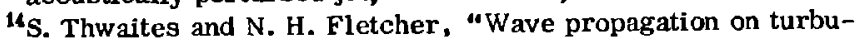
lent jets," Acustica (in press).

${ }^{15}$ I. S. Gradshteyn and I. W. Ryzhik, Tables of integrals, series and products (Academic, New York, 1965), pp. 25-26.

$1 G_{W}$. E. Worman, "Self-sustained non-linear oscillations of medium amplitude in clarinet-like systems," Ph.D. thesis, Case Western Reserve University, Cleveland, OH (1971) [Uni. Microfilms, Ann Arbor (Ref. 71-22869)], pp. 1-154.

${ }^{17}$ A. W. Nolle, "Some voloing adjustments of flue organ pipes," J. Acoust. Soc. Am. 66, 1612-1626 (1979).

${ }^{18}$ A. v. Lüpke, "Utersuchungen an Blockflöten," Akust. Z. 5, 39-46 (1940):

${ }^{19} \mathrm{R}$. Herman, "Observations on the acoustical characteristics of the English flute," Am. J. Phys. 27, 22-29 (1959).

${ }^{20}$ We are grateful to Richard Ward for taking these measurements.

${ }^{21} \mathrm{~N}$. H. Fletcher, "Acoustical correlates of flute performance technique," J. Acoust. Soc. Am. 57, 233-237 (1977).

${ }^{22} \mathrm{~A}$. H. Benade, "Relation of air-column resonances to sound spectra produced by wind instruments," J. Acoust. Soc. Am. 40, 247-249 (1966). 